\title{
Left ventricular long axis disturbances as predictors for thallium perfusion defects in patients with known peripheral vascular disease
}

\author{
M Y Henein, C Anagnostopoulos, S K Das, C O’Sullivan, S R Underwood, D G Gibson
}

\begin{abstract}
Objective-To compare resting long axis echocardiography with adenosine thallium-201 emission tomography in detecting myocardial ischaemic abnormalities in patients before peripheral vascular surgery.

Design-A prospective and blinded preoperative examination of resting left ventricular minor and long axes and myocardial perfusion during adenosine vasodilatation using thallium-201 emission tomography.
\end{abstract}

Setting-A tertiary referral centre for cardiac and vascular disease equipped with invasive, non-invasive, and surgical facilities.

Subjects-65 patients (40 men) with significant peripheral vascular disease, mean (SD) age 63 (10) years, and 21 control subjects of similar age.

Methods-Segments were classified as normal, with fixed or reversible defects according to thallium-201 myocardial perfusion tomography. Systolic long axis abnormalities were either reduced excursion and/or abnormal shortening after A2, and diastolic abnormalities either delayed onset of lengthening $>80 \mathrm{~ms}$ and/or reduced peak lengthening rate $<4.5 \mathrm{~cm} / \mathrm{s}$. Segmental perfusion defects were compared with the equivalent long axes; anteroseptal for septal, inferoseptal for posterior, and lateral for left side giving a total of 195 segments.

Results-Systolic long axis abnormalities predicted fixed thallium defects (sensitivity $86 \%$, specificity $87 \%$, positive predictive value 0.78 , negative predictive value $0.93, p<0.001$ ), and diastolic abnormalities correlated with reversible perfusion defects (sensitivity $90 \%$, specificity $85 \%$, positive predictive value 0.72 , negative predictive value $0.95, p<0.001)$. Echocardiography characteristics of the true and false positive segments were not different in the site or the extent of abnormalities. Conclusion-Systolic long axis abnormalities predict fixed and diastolic reversible thallium perfusion defects in patients with peripheral vascular disease. Ventricular long axis may thus have a value as a screening test before peripheral vascular surgery as well as providing a means of monitoring myocardial perfusion. The high negative predictive values indicate that a negative long axis study makes significant perfusion abnormalities very unlikely in patients with high pretest probability of coronary artery disease. (Heart 1998;79:295-300)

Keywords: peripheral vascular disease; left ventricular long axis; thallium-201; myocardial perfusion scintigraphy

The function of the left ventricular long axis, as assessed from the motion of the mitral ring, is frequently abnormal in patients with coronary artery disease, even in the absence of overt ischaemia. ${ }^{12}$ These disturbances involve the velocity and timing of the ring movement as well as the overall amplitude. They are aggravated by balloon inflation during coronary angioplasty $^{3}$ and improve within 48 hours of a successful procedure. ${ }^{4}$ They are thus likely to represent a potentially reversible functional deficit of the myocardium, which is closely related to the presence of coronary artery stenosis, and which, from the anatomical distribution of the longitudinally directed fibres, ${ }^{5}$ probably involves primarily the subendocardium. The aim of the present investigation was to characterise these disturbances by comparing their frequency, location, and nature with those demonstrated by thallium perfusion imaging. We included a homogenous group of patients with peripheral vascular disease in whom the incidence of coronary artery disease is known to be high, and in whom thallium scanning was clinically indicated. We hoped to gain further insight into the nature of the disturbances of left ventricular long axis function. At a more practical level, we wished to investigate the possible value and limitations of resting long axis echocardiography as a screening test for patients with suspected coronary artery disease.

\section{Patients and methods}

We studied 65 consecutive patients (40 men) with peripheral vascular disease, mean (SD) age 63 (10) years, in whom thallium scanning was required for preoperative cardiac assessment: 19 patients required abdominal aortic aneurysm repair, 13 aorto-iliac, 11 aortobifemoral, 15 femoro-popliteal grafts, and 7 carotid endarterectomy. Pre-existing heart disease was documented in 59 patients: 26 had previous revascularisation (21 coronary artery bypass grafting, 4 coronary angioplasty, 1 coronary stenting), 24 had stable angina without myocardial infarction or revascularisation, and 9 had previous myocardial infarction
Accepted for publication 25 November 1997 
without revascularisation. Three other patients had a history of cerebrovascular accident. No patient had had unstable angina or myocardial infarction in the previous six months, and none had decompensated congestive heart failure or significant chronic obstructive airways disease, and all were in sinus rhythm. No patient underwent any coronary intervention between thallium and echocardiography studies. Twenty one subjects with low likelihood of coronary disease served as controls: 14 were men, mean (SD) age 58 (11) years, and none had peripheral vascular disease, cardiac disease, hypertension or diabetes mellitus. All were in sinus rhythm.

MYOCARDIAL PERFUSION IMAGING

Studies were performed after an overnight fast and 24 hours abstinence from caffeine. Adenosine was infused intravenously $(140 \mathrm{mg} / \mathrm{kg} /$ $\min$ ) for six minutes, together with semisupine bicycle exercise in two minute stages of $25 \mathrm{~W}$, $50 \mathrm{~W}$, and $75 \mathrm{~W}^{6}{ }^{6}$ Thallium-201 (80 MBq) was injected after four minutes and emission tomograms were acquired within five minutes of injection and again after four hours of redistribution. These images were subsequently referred to as stress and redistribution, respectively. A Sopha Medical DS7 camera (BUC, France) was used with a high resolution collimator, 32 projections over $180^{\circ}$, and a body contoured orbit. Energy windows used were $25 \%$ around the $80 \mathrm{keV}$ peak and $20 \%$ around the $167 \mathrm{keV}$ peak. Projections were acquired for 30 seconds for stress and for 40 seconds for redistribution images, giving total acquisition times of 16 minutes and 22 minutes, respectively. Transaxial tomograms were reconstructed by back-projection using a Ramp-Hanning filter with a cut off frequency of 0.75 pixel $^{-1}$, followed by reorientation into oblique tomograms in vertical and horizontal long axis plans and the short axis plan.

Images were interpreted by an experienced observer without knowledge of the echocardiographic findings. For the purposes of comparison, the myocardium was divided into nine segments: basal and apical parts of the anterior, lateral, inferior, and septal walls, together with the apex. A semiquantitative scoring system was used for each segment: 4 , normal; 3 , mild defect; 2, moderate defect; 1 , severe defect; 0 , absent uptake. The scores were assigned by visual estimation of mean activity in each segment, taking into account known normal appearance in each segment (for example, inferior attenuation). Fixed defects were defined as segments with unchanged or worse scores between stress and redistribution. Reversible defects were defined as segments with an improvement of 1 or more between stress and redistribution.

\section{ECHOCARDIOGRAPHY}

Resting Doppler echocardiographic examination was performed in all patients one to two days before surgery, using a Hewlett-Packard echograph with a $2.5 \mathrm{MHz}$ transducer (Andover, Massachusetts, USA). Cross sectional imaging in the left parasternal long axis plane was used to guide $\mathrm{M}$ mode recordings of the left ventricular minor axis, with the cursor by the tips of mitral leaflets and the patient in the semilateral position. The apical four chamber view was used to guide left ventricular long axis $M$ mode recordings, with the cursor positioned at the left and septal angles of the mitral ring, ${ }^{7}$ and the posterior angle from the apical two chamber view. Transmitral Doppler velocities were obtained from the same view with the sample volume at the tips of mitral valve leaflets. The electrocardiogram and a precordial phonocardiogram were also obtained superimposed on each Doppler and $\mathrm{M}$ mode trace. All recordings were made using a paper speed of $100 \mathrm{~mm} / \mathrm{s}$ and traces were later digitised offline to estimate peak velocities. ${ }^{8}$

Left ventricular end diastolic dimension was measured on the minor axis $M$ mode recording from the leading edge of the septal endocardium to that of the posterior wall, at the onset of the Q wave of the electrocardiogram. End systolic dimension was measured in a similar fashion at the time of the A2, the first high frequency component of the second heart sound of the phonocardiogram. The total amplitude of excursion of the mitral ring was measured from the long axis $\mathrm{M}$ mode recording, between the peak apical movement around the second heart sound and peak basal movement at the nadir of the A wave, in late diastole. A wave amplitude was measured as the extent of backward ring motion after the $\mathrm{P}$ wave of the electrocardiogram. Lengthening of the long axis after the $\mathrm{Q}$ wave and shortening after $\mathrm{A} 2$ were also defined as abnormal as neither were observed in the control group, and the extent of the abnormal motion was measured (fig 1). Peak rate of long axis shortening and lengthening were measured from the digitised traces. The time interval from the $Q$ wave of the electrocardiogram to the onset of systolic shortening was measured together with that from A2 of the phonocardiogram to the onset of lengthening. Peak transmitral early and late filling velocities were also measured from the pulsed Doppler trace.

For each long axis trace, two diastolic and two systolic measurements (outside the 95\% confidence intervals (CI) of normal) at each of the three left ventricular sites were assessed. The systolic parameters were a reduction of the total extent of shortening to less than $1.0 \mathrm{~cm}$ and abnormal prolongation of shortening after aortic valve closure by more than $1 \mathrm{~mm}$. The diastolic parameters were a delay in the onset of lengthening by more than $80 \mathrm{~ms}$ after A2, and an early diastolic peak lengthening rate of less than $4.5 \mathrm{~cm} / \mathrm{s}$. For a segment to be considered abnormal at least one of the two criteria had to be fulfilled.

COMPARISON OF THE TWO TECHNIQUES

In the 65 patients segmental perfusion was assessed by thallium as being normal or having either a fixed or a reversible defect. This was compared with the equivalent long axes: anteroseptal for septal; inferoseptal for posterior; and lateral for left side. We combined apical and basal perfusion defects and did not 
Table 1 Reproducibility of long axis variables

\begin{tabular}{|c|c|c|}
\hline \multirow[b]{2}{*}{ Variables } & \multicolumn{2}{|c|}{ Coefficient of variation (\%) } \\
\hline & Intraobserver & Interobserver \\
\hline Total excursion & 3.2 & 4.0 \\
\hline $\begin{array}{l}\text { Shortening during isovolumic } \\
\text { relaxation }\end{array}$ & 11.5 & 15.0 \\
\hline Peak shortening rate & 4.2 & 5.4 \\
\hline Peak lengthening rate & 5.0 & 6.0 \\
\hline $\mathrm{Q}$ wave onset of shortening & 6.5 & 7.0 \\
\hline A 2 onset of lengthening & 5.5 & 6.0 \\
\hline
\end{tabular}

consider the apex to be an independent segment. Therefore, the two techniques were compared on the basis of three segments: anteroseptal, inferoseptal, and lateral, giving a total of 195 segments in the 65 patients. The characteristics of the true and false positive results were compared in terms of site, shortening and lengthening velocity, and timing.

LONG AXIS REPRODUCIBILITY

The reproducibility of the digitised process was assessed in a sample of 20 patients from duplicate determination of long axis excursion, peak velocities, and timing with respect to $Q$ wave and A2. Within and between observer values were determined independently. Reproducibility was assessed as root mean square (RMS) difference between duplicate measurements, and the corresponding value of coefficient of variation as the ratio RMS difference:absolute value (table 1 ).

\section{STATISTICS}

Comparisons between echocardiographic parameters in the different patient groups as classified by myocardial perfusion imaging were made using an unpaired Student's $t$ test.
Abnormal values in patients were identified as being outside the $95 \% \mathrm{CI}$ of the normal range when they were higher or lower than 2 SD from the corresponding mean in the normal group. Sensitivity, specificity, and predictive values were calculated using thallium perfusion results as the standard. A $\chi^{2}$ test was used to compare the incidence of echocardiographic and perfusion imaging abnormalities.

\section{Results}

MYOCARDIAL PERFUSION IMAGING

Adenosine infusion was uncomplicated and good quality tomograms were acquired in all patients. Heart rate rose from 74 (15) to 100 (23) beats/min and systolic blood pressure from 145 (20) to 165 (20) $\mathrm{mm} \mathrm{Hg}$. Five patients had normal stress tomograms. Of the 44 patients with known coronary artery disease but without a history of myocardial infarction, four had an isolated fixed defect, 19 had an isolated reversible defect, 14 patients had both, and 3 had no significant perfusion defect. Of the 9 patients with known coronary artery disease and prior infarction, all had fixed defects and 7 had additional reversible defects. Twelve of the 15 patients with normal myocardial perfusion had no history of coronary artery disease.

\section{ECHOCARDIOGRAPHY}

Satisfactory echocardiograms showing left ventricular minor and all three long axes were obtained in all 65 patients. Left ventricular minor axis dimension at end diastole was abnormally increased in the patient groups, and to a greater extent in patients with fixed compared to those with isolated reversible defects (table 2). Mitral flow velocities showed

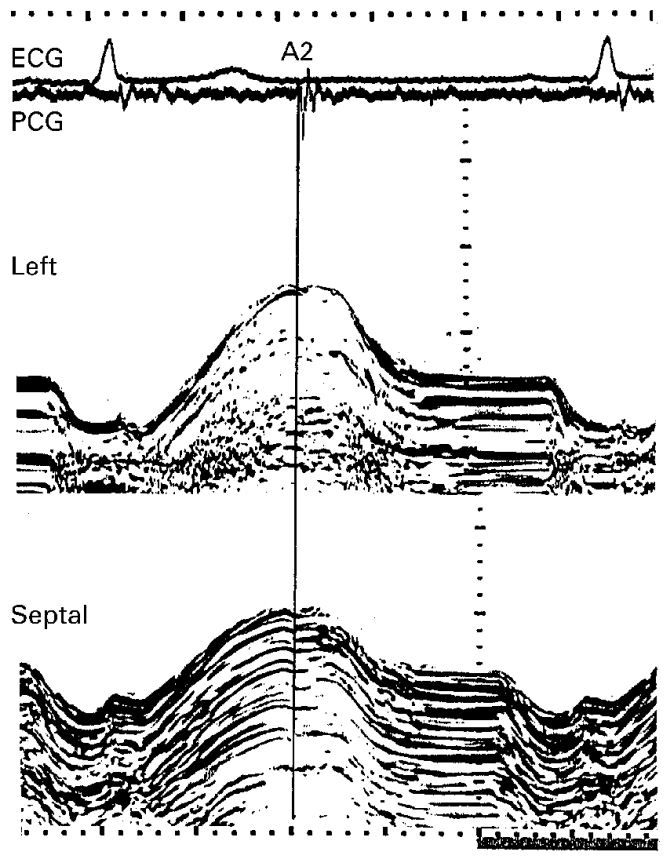

Control

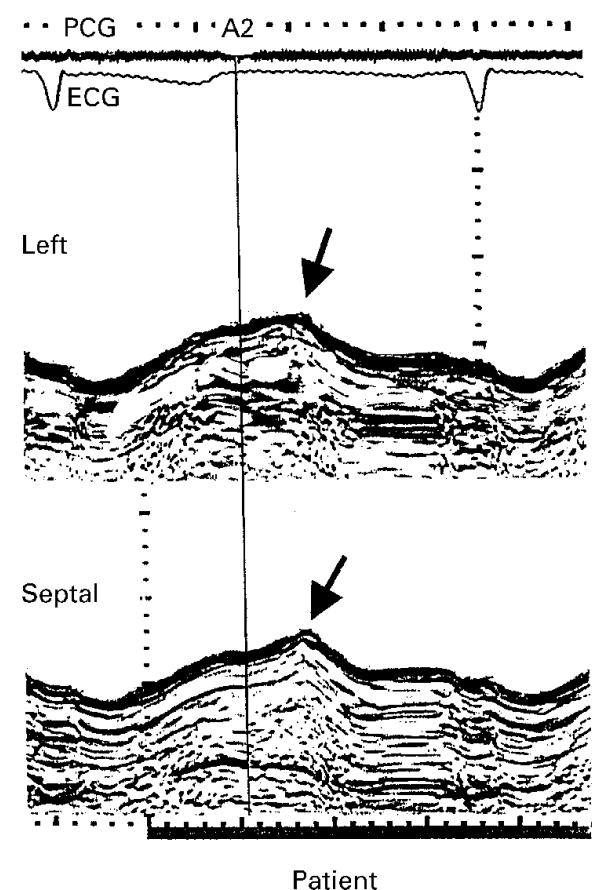

Patient

Figure 1 An example of long axis recording of the left and septal sites from a control subject (left) and a patient (right). In systole, the extent of total long axis excursion is reduced to $7 \mathrm{~mm}$ and there is abnormal shortening after A2 (arrow). In diastole, the onset of lengthening is delayed and early diastolic lengthening velocity reduced. 
Table 2 Left ventricular minor axis and mitral Doppler

\begin{tabular}{|c|c|c|c|c|c|c|c|}
\hline & & \multirow[b]{2}{*}{$\begin{array}{l}\text { Normal } \\
(n=21)\end{array}$} & \multicolumn{5}{|c|}{ Thallium perfusion defect } \\
\hline & & & \multicolumn{2}{|l|}{$\begin{array}{l}\text { Reversible } \\
(n=24)\end{array}$} & \multicolumn{2}{|c|}{$\begin{array}{l}\text { Fixed } \\
(n=8)\end{array}$} & $\begin{array}{l}\text { Mixed } \\
(n=18)\end{array}$ \\
\hline \multicolumn{8}{|l|}{ Minor axis } \\
\hline \multirow{2}{*}{\multicolumn{2}{|c|}{$\begin{array}{l}\text { End diastolic dimension }(\mathrm{cm}) \\
\text { End systolic dimension }(\mathrm{cm})\end{array}$}} & $4.8(0.5)$ & \multicolumn{2}{|c|}{$5.4(0.6)^{\star \star \star}$} & \multicolumn{2}{|c|}{$6.2(0.8)^{\star \star \star / \star \star}$} & $5.7(0.8)^{\star \star \star}$ \\
\hline & & \multirow{2}{*}{$3.3(0.5)$} & \multicolumn{2}{|c|}{$3.8(0.7)^{\star \star}$} & \multicolumn{2}{|c|}{$4.7(1.2)^{\star \star \star / \star}$} & $4.2(1.0)^{\star \star \star}$ \\
\hline \multicolumn{7}{|l|}{ Transmitral Doppler } & \\
\hline Early diastolic velocity $(\mathrm{m} / \mathrm{s})$ & & $0.7(0.1)$ & \multicolumn{2}{|l|}{$0.6(0.2)$} & \multicolumn{2}{|c|}{$0.5(0.3)$} & $0.6(0.2)$ \\
\hline Late diastolic velocity $(\mathrm{m} / \mathrm{s})$ & & $0.5(0.1)$ & \multicolumn{2}{|l|}{$0.7(0.2)$} & \multicolumn{2}{|c|}{$0.6(0.3)$} & $0.8(0.2)$ \\
\hline \multicolumn{8}{|c|}{${ }^{\star} \mathrm{p}<0.05 ;{ }^{\star \star} \mathrm{p}<0.01 ;{ }^{\star \star \star} \mathrm{p}<0.001$ first: $v$ normals; second: $v$ reversible. } \\
\hline $\begin{array}{l}\text { Table } 3 \text { Incidence of systolic } \\
\text { fixed and reversible thallium } p\end{array}$ & and & $\begin{array}{l}\text { diastolic l } \\
\text { sion defect }\end{array}$ & long axis abno & & alities i & the segm & nents showing \\
\hline Thallium/echocardiography & $\begin{array}{l}\text { Both } \\
\text { abn }\end{array}$ & rmal & $\begin{array}{l}\text { Thallium } \\
\text { abnormal }\end{array}$ & & hormal & $\begin{array}{l}\text { Both } \\
\text { normal }\end{array}$ & $p$ value \\
\hline Fixed/systolic & & & & & & & \\
\hline Left & 9 & & 1 & 7 & & 48 & $<0.001$ \\
\hline Septal & 25 & & 2 & 7 & & 31 & $<0.001$ \\
\hline Posterior & 20 & & 6 & 1 & & 38 & $<0.02$ \\
\hline Total & 54 & & 9 & 15 & & 117 & $<0.001$ \\
\hline Fixed (total)/diastolic & & & & & & & \\
\hline Left & 9 & & 6 & 25 & & 25 & NS \\
\hline Septal & 10 & & 2 & 30 & & 23 & NS \\
\hline Posterior & 9 & & 5 & 25 & & 26 & NS \\
\hline Total & 28 & & 13 & 80 & & 72 & NS \\
\hline Fixed (isolated)/diastolic & & & & & & & \\
\hline Left & 4 & & 3 & 15 & & 43 & NS \\
\hline Septal & 6 & & 4 & 16 & & 39 & NS \\
\hline Posterior & 4 & & 7 & 13 & & 41 & NS \\
\hline Total & 14 & & 14 & 44 & & 123 & NS \\
\hline Reversible/systolic & & & & & & & \\
\hline Left & 3 & & 5 & 17 & & 40 & NS \\
\hline Septal & 10 & & 4 & 29 & & 22 & NS \\
\hline Posterior & 8 & & 5 & 25 & & 27 & NS \\
\hline Total & 21 & & 14 & 71 & & 89 & NS \\
\hline Reversible (total)/diastolic & & & & & & & \\
\hline Left & 22 & & 0 & 14 & & 29 & $<0.001$ \\
\hline Septal & 39 & & 4 & 5 & & 17 & $<0.001$ \\
\hline Posterior & 26 & & 8 & 6 & & 25 & $<0.001$ \\
\hline Total & 87 & & 12 & 25 & & 71 & $<0.001$ \\
\hline Rever (isolated)/diastolic & & & & & & & \\
\hline Left & 12 & & 0 & 13 & & 40 & $<0.001$ \\
\hline Septal & 23 & & 2 & 5 & & 35 & $<0.001$ \\
\hline Posterior & 18 & & 4 & 3 & & 40 & $<0.001$ \\
\hline Total & 53 & & 6 & 21 & & 115 & $<0.001$ \\
\hline
\end{tabular}

Table 4 Diagnostic tests and predictive values

\begin{tabular}{lllll}
\hline Thallium/echocardiography & Sensitivity & Specificity & $\begin{array}{l}\text { Positive } \\
\text { predictive value }\end{array}$ & $\begin{array}{l}\text { Negative } \\
\text { predictive value }\end{array}$ \\
\hline Fixed/systolic & $86 \%$ & $87 \%$ & 0.78 & 0.93 \\
Fixed/diastolic & $77 \%$ & $46 \%$ & 0.25 & 0.9 \\
Reversible/diastolic & $88 \%$ & $73 \%$ & 0.78 & 0.86 \\
Revers (isolated)/diastolic & $90 \%$ & $85 \%$ & 0.72 & 0.95 \\
\hline
\end{tabular}

Table 5 Comparison between values in the two groups with abnormal long axis function

\begin{tabular}{llll}
\hline Long axis abnormalities & True positive & False positive & p value \\
\hline Total excursion $(\mathrm{cm})$ & $0.9-0.65(\mathrm{n}=40)$ & $0.64-0.9(\mathrm{n}=12)$ & NS \\
Abnormal shortening after A2 $(\mathrm{mm})$ & $1-3(\mathrm{n}=25)$ & $1-2.8(\mathrm{n}=5)$ & NS \\
Peak lengthening rate $(\mathrm{cm} / \mathrm{s})$ & $4.5-2.5(\mathrm{n}=72)$ & $4.5-2.7(\mathrm{n}=18)$ & NS \\
Delayed onset of lengthening $(\mathrm{ms})$ & $80-120(\mathrm{n}=60)$ & $80-125(\mathrm{n}=20)$ & NS \\
\hline
\end{tabular}

$\mathrm{n}$, number of segments showing each abnormality.

no significant correlation with thallium abnormalities (table 2). Long axis segments were considered abnormal if they showed one or more of the two systolic (reduced total excursion or abnormal shortening after A2) or two diastolic (delayed onset or reduced peak lengthening rate) abnormalities.

Segmental comparison in individual patients Table 3 shows the incidence of abnormal systolic and diastolic long axis values in each individual segment, classified as normal or as having a fixed, a reversible or mixed perfusion defect. When both fixed and reversible defects affected the same segment they were classified in both groups. Table 3 also shows the direct comparison between thallium perfusion and long axis echo abnormalities in each of the 195 segments studied. There was a strong association between fixed thallium defects and systolic long axis abnormalities and between reversible thallium defects and isolated diastolic long axis abnormalities. Table 4 shows the corresponding values of sensitivity, specificity, and predictive accuracy. For both comparisons, the false positive rate was significantly higher than the false negative rate, which was very low for both comparisons. To assess the possibility that inappropriate cut off levels were used, the echocardiography characteristics of true positive versus false positive segments were compared. There was no difference in site or in the extent of velocity or time interval between the two, for either systolic or diastolic abnormalities (table 5). There was no association between fixed thallium and diastolic long axis or between reversible thallium and systolic long axis abnormalities.

CLINICAL OUTCOME

No patient experienced any clinically apparent cardiac complications, and routine postoperative ECG and assessment of cardiac enzymes showed no evidence of myocardial infarction. In the 65 patients there were two postoperative deaths ( $3 \%$ of the operated patients), one a month after surgery from cerebrovascular accident and one from renal failure.

\section{Discussion}

This study has confirmed previous ones in demonstrating that mitral flow velocities were effectively normal in a group of patients with a high incidence of chronic coronary artery disease. Mean values of end diastolic and end systolic cavity dimensions were increased, but this relation was of no value in predicting the result of thallium perfusion scanning in individual patients. When ventricular long axis function was considered, the position was different, with systolic long axis abnormalities correlating with fixed defects and diastolic abnormalities with reversible defects on thallium perfusion imaging. Clearly, the two techniques were not interchangeable. The ability of long axis echocardiography to localise rather than simply detect defects was limited compared with thallium imaging or standard stress echocardiography, as only three rather than nine potential sites could be distinguished. In addition, long axis echocardiography was unable to make the clinically important distinction between isolated fixed defects and the combination of fixed with associated reversible ischaemia. Nevertheless, resting long axis echocardiography has the advantage that complete recordings can be obtained within three minutes. This, in combination with its low false negative rate, gives it potential value as a screening test. In addition, it can readily be applied to the right ventricle, which is not easily accessible to routine thallium imaging. In common with stress echocardiography, it avoids the use of radiation exposure. It 
also lends itself to making repeated observations in the same patient, and so can be used for observing time related effects of drugs or other haemodynamic manoeuvres on potentially ischaemic myocardium. ${ }^{9}$ The combination of a relatively long test time, and the provocation of overt ischaemia by the investigation itself makes both thallium scanning and standard stress echocardiography unsuitable for this application in patients with suspected myocardial ischaemia. ${ }^{1011}$

\section{LIMITATIONS}

The thallium scan and echocardiograms were not performed simultaneously, so that real differences in the state of the myocardium may have occurred between the two. There is little information in the literature about the extent of such changes in otherwise stable patients. For ethical reasons, we were unable to define our own criteria of normality for thallium scanning as part of the study design, and on the same grounds did not perform coronary arteriography in the elderly group of apparently normal subjects used as controls. We considered, and rejected, the alternative possibility of using a group of patients with symptoms of angina but normal coronary arteriograms. These patients are usually younger and predominantly female; older men who fit these criteria are uncommon, and likely to have other underlying diagnoses. We made our comparison within a group of patients with a high incidence of coronary artery disease in whom thallium scanning was clinically indicated. We elected to do so, both to reflect normal practice and to avoid the spuriously high levels of predictive accuracy that may arise when the obviously normal and obviously abnormal are compared directly. Our results cannot therefore be assumed to apply in other circumstances, such as left ventricular hypertrophy or cardiomyopathy. We were unable to extend our results to the right ventricle as thallium perfusion would not have been a reliable standard of comparison. We were also unable to correlate the results of thallium imaging and echocardiography to clinically apparent operative risk as the incidence of cardiac complications was low.

An unexpected finding was that long axis false positive rates were consistently more than twice the false negative rates compared with thallium for both systolic and diastolic abnormalities. The mean value of all measurements of long axis function was similar in true and false positives, so agreement would not have improved by adopting echocardiography criteria stricter than those based on the $95 \%$ CI of normal, suggesting that the problem was not a simple one of sensitivity. At the same time, the very low false negative rate strongly suggests that random error cannot be invoked. We would suggest, therefore, that these apparently discordant findings provide evidence of an additional population of patients, not detected by thallium, in whom disturbances to long axis function were just as severe as those with clear cut perfusion defects. We have no definite information as to their nature, but the anatomical localisation of longitudinally directed fibres suggests that isolated subendocardial ischaemia or infarction might lead to this characteristic picture.

The basis of the correlation between systolic long axis abnormalities, particularly reduced overall amplitude of motion, and fixed thallium defects seems clear, as both are likely to reflect the presence of old myocardial infarction. The connection between diastolic long axis disturbances and reversible thallium defects does not appear so straightforward. The long axis was abnormal at rest whereas reversible thallium defects became apparent only with stress, strongly suggesting that unlike the position with fixed defects, the two techniques are not detecting the same pathological entity. Diastolic long axis disturbances are strikingly aggravated during pharmacological stress, with tension persisting until the onset of succeeding atrial or even ventricular systole. ${ }^{12}$ It is possible, therefore, that this prolonged tension development during pharmacological stress may directly interfere with coronary flow. This might explain why local thallium uptake, and thus myocardial perfusion, actually falls rather than simply failing to increase with myocardial stimulation. It may also be significant that long axis disturbances occur in uncomplicated left bundle branch block regardless of the presence of coronary artery disease, and that this condition is commonly associated with false positive thallium perfusion defects. ${ }^{13} 14$

\section{CONCLUSIONS}

Our results suggest that in patients with known or suspected coronary artery disease, it may be useful to add resting long axis measurements to the standard echocardiographic examination. This is particularly the case in patients with peripheral vascular disease in whom standard exercise testing is not appropriate. In such patients, in whom the incidence of coronary artery disease is high, resting disturbances may suggest the need for further investigation by thallium perfusion imaging, stress echocardiography, or coronary arteriography. Conversely, if long axis function is normal, the incidence of corresponding thallium perfusion abnormalities is very low. When considered with previous studies, ${ }^{412}$ our results are compatible with the idea that diastolic long axis abnormalities represent a low level of resting myocardial ischaemia occurring in patients with coronary artery disease, which, with stress, will lead to a reversible thallium abnormality. Long axis echocardiography may thus provide a means of studying the natural history of these disturbances in more detail. In particular, it might be possible to gain further information as to their overall clinical significance within the spectrum of myocardial ischaemia by exploiting the clear differences between thallium perfusion imaging and long axis echocardiography. More information is needed about the natural history of thallium negative/long axis positive segments. In addition, the rapid time response of long axis echocardiography might, for the basis of methods, define the acute effects of interventions or drugs in a way not possible with more established techniques. 
1 Henein MY. Ventricular long axis function in coronary artery disease. Imperial College, National Heart \& Lung

Institute, London University. PhD thesis, 1996.
2 Henein MY, Patel DJ, Fox KM, et al. Asynchronous left ventricular wall motion in unstable angina. Int $\mathcal{F}$ Cardio 1996;59:37-45.

3 Henein MY, O'Sullivan C, Davies S, et al. Effects of acute coronary occlusion and previous ischaemic injury on left ventricular wall motion in humans. Heart 1997;77:338-45.

4 Henein MY, Priestley K, Davarashvili T, et al. Early changes in left ventricular subendocardial function after successful coronary angioplasty. Br Heart f 1993;69:501-6

5 Williams PL, Warwick R. Myocardial architecture. In: Gray's anatomy. 36th ed. Edinburgh: Churchill Livingstone, 1980:657-9.

6 Pennell DJ, Mavrogeni S, Forbat SM, et al. Adenosine combined with exercise for myocardial perfusion imaging. $f \mathrm{Am}$ Coll Cardiol 1995;25:1300-9.

7 Jones CJH, Raposo L, Gibson DG. Functional importance of the long axis dynamics of the human left ventricle. $\mathrm{Br}$ of the long axis dynamics

8 Gibson DG, Brown D. Measurement of instantaneous left ventricular dimension and filling rate in man using echocardiography. Br Heart f 1973;35:1141-9.
9 Henein MY, Das SK, O'Sullivan C, et al. Effect of acute alterations in afterload on left ventricular function in
patients with combined coronary artery and peripheral patients with combined coronary artery
vascular disease. Heart 1996;75:151-8

10 Brown KA, Rowen M. Extent of jeopardised viable myocardium determined by myocardial perfusion imaging best predicts perioperative cardiac events in patients undergoing noncardiac surgery. f Am Coll Cardiol 1993;21:325-30.

11 Langan EM III, Youkey JR, Franklin DP, et al. Dobutamine stress echocardiography for risk assessment before aortic surgery. F Vasc Surg 1993;18:905-13.

12 Henein MY, O'Sullivan CA, Koh TW, et al. Dobutamine stress echocardiography reveals early impairment of diastolic ventricular function in coronary artery disease [abstract]. Circulation 1996;94:I-679.

13 Xiao HB, Roy C, Gibson DG. Correlation between the sequence of ventricular wall motion and ORS morphology in patients with bundle branch block. Eur f Clin Pacing Electrophysiol 1995;5:10-17.

14 Rothbart RM, Beller GA, Watson DD, et al. Diagnostic accuracy and prognostic significance of quantitative accuracy and prognostic significance of quantitative
thallium 201 scintigraphy in patients with left bundle thallium 201 scintigraphy in patients with left bundle
branch block. American fournal of Non-invasive Cardiology 1987;1:197-205. 